\title{
Direct preferences for wealth, the risk premium puzzle, growth, and policy effectiveness ${ }^{\text {is }}$
}

\author{
Liutang Gong ${ }^{\mathrm{b}, \mathrm{c}}$, Heng-fu Zou ${ }^{\mathrm{a}, \mathrm{b}, \mathrm{c}, *}$ \\ ${ }^{a}$ Development Research Group, The World Bank, 1818 H Street, NW, Washington, \\ DC 20433, USA \\ ${ }^{\mathrm{b}}$ Institute for Advanced Study, Wuhan University, China \\ 'Guanghua School of Management, Peking University, China \\ Received 4 November 1997; accepted 5 May 2000
}

\begin{abstract}
In this paper, we consider social status, the spirit of capitalism, fiscal policies, and asset pricing in a stochastic model of growth. With specific assumptions on the production technology, preferences, and stochastic shocks, we derive the explicit solutions to the growth rates of consumption and savings and equilibrium returns on all assets. We further demonstrate how fiscal policies, the spirit of capitalism, and stochastic shocks affect growth, asset pricing, and welfare. (C) 2002 Elsevier Science B.V. All rights reserved.
\end{abstract}

JEL classification: E0; G1; H0; O0

Keywords: Social status; The spirit of capitalism; Fiscal policies; Asset pricing; Stochastic growth

\section{Introduction}

In neoclassical growth models wealth accumulation is often taken to be solely driven by one's desire to increase consumption rewards. The representative

\footnotetext{
${ }^{7}$ We thank a referee for very constructive comments. Of course, we are responsible for any remaining errors.

* Correspondence address. World Bank, MC2-611, 1818 H St. NW, Washington, DC 20433, USA.
} 
agent chooses a consumption path to maximize his discounted utility, which is defined only on consumption. This motive is important for wealth accumulation. It is, however, not the only motive. Because man is a social animal, he also accumulates wealth to gain prestige, social status, and power in the society; see Frank (1985), Cole et al. (1992, 1995), Fershtman and Weiss (1993), Zou (1994, 1995), Bakshi and Chen (1996), and Fershtman et al. (1996). Earlier contributions include Duesenberry (1948), Kurz (1968), and Spence (1974). In these wealth-is-status models, the representative agent has direct preferences for wealth and accumulates wealth not only for consumption but also for wealthinduced status. Mathematically, in light of the new perspective, the utility function can be defined on both consumption, $c$, and wealth, $W: u\left(c_{t}, W_{t}\right)$. Another interpretation of these models is in line with the spirit of capitalism in the sense of Weber (1958) and Keynes (1971): capitalists accumulate wealth for the sake of wealth. To cite Weber (1958):

Man is dominated by making of money, by acquisition as the ultimate purpose of his life. Economic acquisition is no longer subordinated to man as the means for the satisfaction of his material needs. This reversal relationship, so irrational from a naive point of view, is evidently a leading principle of capitalism.

Using the wealth-is-status and the-spirit-of-capitalism models, many authors have tried to explain growth, savings, and asset pricing. Cole et al. (1992) have demonstrated how the presence of social status leads to multiple equilibria in long-run growth. Zou (1994, 1995) has studied the spirit of capitalism and long-run growth and showed that a strong capitalist spirit can lead to unbounded growth of consumption and capital even under the neoclassical assumption of production technology. Bakshi and Chen (1996) have explored empirically the relationship between the spirit of capitalism and stock market pricing and offered an attempt towards the resolution of the equity premium puzzle in Mehra and Prescott (1985). They have shown that when investors care about status they will be more conservative in risk taking and more frugal in consumption spending. Furthermore, stock prices tend to be more volatile with the presence of the spirit of capitalism.

On the other hand, Eaton (1981), Turnovsky (1993, 1995), Grinols and Turnovsky $(1993,1994)$, and Obstfeld (1994) have introduced stochastic tax and stochastic government expenditure into the continuous-time growth and assetpricing models. Under specific assumptions on the production technology, preferences, and stochastic shocks, they have derived explicit solutions to the growth rates of consumption and savings and equilibrium returns on assets. But these continuous-time stochastic growth models have not explicitly considered the role of social status and the spirit of capitalism in capital accumulation, asset pricing, and growth.

In this paper, we integrate these two trends of growth and asset-pricing literature and consider social status, fiscal policies, and asset pricing in a stochastic model of growth. With specific assumptions on the production 
technology, preferences, and stochastic shocks, we derive the explicit solutions to the growth rates of consumption and savings and equilibrium returns on all assets. We further demonstrate how fiscal policies, social status, the spirit of capitalism, and stochastic shocks affect economic growth and asset pricing.

The paper is organized as follows: in Section 2, we present a modified growth and asset-pricing framework as in Turnovsky (1995) and Bakshi and Chen (1996). In Section 3, we derive the optimal conditions for macroeconomic equilibrium. In Section 4, using a specific utility function, we present explicit solutions to the consumption-wealth ratio, the mean growth rate of the economy, and the expected real return on bonds and capital. In Section 5, we discuss the effects of stochastic shocks and fiscal policies on the economy. In Section 6, we discuss the effects of the concern for social status or the spirit of capitalism on asset pricing and growth. We conclude the paper in Section 7.

\section{The model}

Along with Eaton (1981) and Turnovsky (1995), we assume output $Y$ and government expenditure $G$ to be proportional to the mean-level output, i.e.

$$
\begin{aligned}
& \mathrm{d} Y=\alpha K \mathrm{~d} t+\alpha K \mathrm{~d} y, \\
& \mathrm{~d} G=g \alpha K \mathrm{~d} t+\alpha K \mathrm{~d} z .
\end{aligned}
$$

Eq. (1) asserts that the accumulated flow of output over the period $(t, t+\mathrm{d} t)$, given by the right-hand side of this equation, consists of two components. The deterministic component is described as the first term on the right hand, which is the firm's production technology and has been specified as a linear production function. The second part is the stochastic component, which can be viewed as the shock to the production and assumed to be temporally independent, normally distributed, and

$$
E(\mathrm{~d} y)=0, \quad \operatorname{Var}(\mathrm{d} y)=\sigma_{y}^{2} \mathrm{~d} t .
$$

In Eq. (2), the deterministic part of government expenditure is expressed in terms of a fraction of mean output, and government expenditure has the stochastic shock $\mathrm{d} z$. It is further assumed that $\mathrm{d} z$ is temporally independent, normally distributed, and

$$
E(\mathrm{~d} z)=0, \quad \operatorname{Var}(\mathrm{d} z)=\sigma_{z}^{2} \mathrm{~d} t .
$$

Following Turnovsky (1995), it is assumed that there are two assets in the economy: government bonds, $B$, and the capital stock, $K$. If the inflation rate is stochastic as in Fischer (1975), the return on government bonds $B$ will also follow a stochastic process. Without providing much detail on the derivations, it 
is postulated, as in Turnovsky (1995) that the stochastic real rate of return on bonds, $\mathrm{d} R_{B}$, over a period $\mathrm{d} t$, is given by

$$
\mathrm{d} R_{B}=r_{B} \mathrm{~d} t+\mathrm{d} u_{B},
$$

where $r_{B}$ and $\mathrm{d} u_{B}$ will be determined endogenously in the macroeconomic equilibrium.

Turning to the second asset, capital, and using the production technology in Eq. (1), the stochastic real rate of return on capital is

$$
\mathrm{d} R_{K}=\frac{\mathrm{d} Y}{K}=\alpha \mathrm{d} t+\alpha \mathrm{d} y=r_{K} \mathrm{~d} t+\mathrm{d} u_{K}
$$

Thus, wealth $W(t)$ is the sum of the holdings of $B(t)$ and $K(t)$, i.e.,

$$
W(t)=B(t)+K(t)
$$

Let $n_{B}$ and $n_{K}$ denote the fractions of wealth invested in bonds and capital, respectively, i.e.,

$$
n_{B}=\frac{B(t)}{W(t)}, \quad n_{K}=\frac{K(t)}{W(t)}
$$

and $n_{B}+n_{K}=1$.

We may assume that, without any loss of generality, taxes are levied on capital income and consumption, namely,

$$
\mathrm{d} T=\left(\tau r_{K} K+\tau_{c} c\right) \mathrm{d} t+\tau^{\prime} K \mathrm{~d} u_{K}=\left(\tau \alpha K+\tau_{c} c_{t}\right) \mathrm{d} t+\tau^{\prime} \alpha K \mathrm{~d} y,
$$

where $\tau, \tau^{\prime}$ are the tax rates on the deterministic component of capital income and the stochastic capital income, respectively, and $\tau_{c}$ is the tax rate on consumption. The introduction of a consumption tax into the model is new. As shown later, consumption tax impacts on economic growth in the wealth-isstatus or the spirit-of-capitalism model. In the traditional setup with the utility defined only on consumption, a consumption tax has no long-run effect on growth and wealth accumulation, it only crowds out private consumption.

Now, the representative agent chooses the consumption-wealth ratio, $c / W$, and the portfolio shares, $n_{B}$ and $n_{K}$, to maximize his expected utility subject to the budget constraint, i.e.,

$$
\max E \int_{0}^{\infty} u\left(c_{t}, W_{t}\right) \mathrm{e}^{-\beta t} \mathrm{~d} t
$$

subject to

$$
\begin{aligned}
& \mathrm{d} W_{t}=\left(n_{B} W_{t} r_{B}+n_{K} W_{t}(1-\tau) r_{K}-\left(1+\tau_{c}\right) c_{t}\right) \mathrm{d} t+W_{t} \mathrm{~d} w \\
& n_{B}+n_{K}=1,
\end{aligned}
$$


where $\beta$ is the time discount rate. The initial stocks of bonds and capital are given by $B(0)$ and $K(0)$, respectively. In addition,

$$
\mathrm{d} w=n_{B} \mathrm{~d} u_{B}+n_{K}\left(1-\tau^{\prime}\right) \mathrm{d} u_{K}
$$

The inclusion of total wealth as an argument in the utility function has been done in many deterministic models mentioned in our introductory section, and its presence in a stochastic model appears only in Bakshi and Chen (1996) in the context of stock-market pricing. But recently Turnovsky (1995), Grinols (1996), and Grinols and Turnovsky (1996) have included real balances (or liquidity services), which are a component of wealth, in the utility function in their studies of fiscal and especially monetary policies in stochastic models. Since we will consider a real economy here, real balances are not in the picture. It is natural to extend the model to a monetary economy and take real balances as a part of total wealth. This is clearly a direction for further research. It will be interesting to compare the results obtained here with the ones with money.

\section{Macroeconomic equilibrium}

As in Turnovsky (1995), the economic system in equilibrium determines the rates of consumption and savings, the value of returns on all assets, and the economic growth rate.

The exogenous variables include the preference parameters, technology parameters, and government fiscal policies including government expenditure $g$, tax rates $\tau, \tau^{\prime}$, and $\tau_{c}$. The exogenous stochastic processes consist of government expenditure, $\mathrm{d} z$, and productivity shocks, $\mathrm{d} y$, which are taken to be mutually uncorrelated. The remaining stochastic disturbances - real rates of returns on bonds, $\mathrm{d} u_{B}$, and total wealth, $\mathrm{d} w$, are both endogenous and will be determined by the economic system. The remaining endogenous variables include the following: the consumption-wealth ratio, $c / W$, the mean growth rate of the economy, the expected real returns on two assets, $r_{B}$, and $r_{K}$, respectively, and the corresponding portfolio shares $n_{B}$ and $n_{K}$.

To solve the agent's optimization problem, we introduce the value function

$$
V(W(t), t)=\max E_{t} \int_{t}^{\infty} u\left(c_{s}, W_{s}\right) \mathrm{e}^{-\beta s} \mathrm{~d} s
$$

subject to (6) and (7).

Define

$$
V(W, t)=\mathrm{e}^{-\beta t} X(W, t) .
$$


Proposition 3.1. The first-order conditions for the optimization problem can be written as follows:

$$
\begin{aligned}
& \frac{\partial u(c, W)}{\partial c}=\left(1+\tau_{c}\right) X_{W}, \\
& \left(r_{B} X_{W} W-\eta\right) \mathrm{d} t+\operatorname{cov}\left(\mathrm{d} w, \mathrm{~d} u_{B}\right) X_{W W} W^{2}=0, \\
& \left((1-\tau) r_{K} X_{W} W-\eta\right) \mathrm{d} t+\operatorname{cov}\left(\mathrm{d} w,\left(1-\tau^{\prime}\right) \mathrm{d} u_{K}\right) X_{W W} W^{2}=0, \\
& n_{B}+n_{K}=1,
\end{aligned}
$$

where $\eta$ is the Lagrangian multiplier associated with the portfolio selection constraint (7). Furthermore, the optimal solutions of the problem must satisfy the Bellman equation

$$
\begin{aligned}
& u(c, W)-\beta X(W, t)+X_{t}(W, t)+\left(\rho-\left(1+\tau_{c}\right) \frac{c}{W}\right) W X_{W} \\
& +\frac{1}{2} \sigma_{w}^{2} W^{2} X_{W W}=0
\end{aligned}
$$

where $\rho=n_{B} r_{B}+n_{K}(1-\tau) r_{K}$, and it is the expected net-of-tax return on total asset holdings.

See the details of the proof in Appendix A.

Condition (9) asserts that in the equilibrium the marginal utility of consumption must equal the marginal utility of wealth; conditions (10) and (11) are the asset pricing relationships; condition (12) is the portfolio selection constraint; and Eq. (13) is the Bellman equation, from which we will solve the value function $X(W, t)$.

In order to determine the full equilibrium system, we follow Turnovsky (1995) in discussing government behavior. Eqs. (2) and (5) describe government expenditure policy and tax policies, both of which are proportional to current output. In the absence of lump-sum taxation, government budget constraint can be described as

$$
\mathrm{d} B=B \mathrm{~d} R_{B}+\mathrm{d} G-\mathrm{d} T
$$

From (2) and (5), this can be written in the form

$$
\frac{\mathrm{d} B}{W}=\frac{B}{W} \mathrm{~d} R_{B}+(g-\tau) \alpha \frac{K}{W} \mathrm{~d} t-\tau_{c} \frac{c}{W} \mathrm{~d} t+\alpha \frac{K}{W} \mathrm{~d} z-\tau^{\prime} \alpha \frac{K}{W} \mathrm{~d} y
$$


i.e.,

$$
n_{B} \frac{\mathrm{d} B}{B}=\left(r_{B} n_{B}-\tau_{c} \frac{c}{W}+\alpha(g-\tau) n_{K}\right) \mathrm{d} t+n_{B} \mathrm{~d} u_{B}+\alpha n_{K} \mathrm{~d} z-\tau^{\prime} \alpha n_{K} \mathrm{~d} y .
$$

For the equilibrium in the product market, we have

$$
\mathrm{d} K=\mathrm{d} Y-c \mathrm{~d} t-\mathrm{d} G
$$

where $G$ follows the stochastic process of Eq. (2). Now, we have

Proposition 3.2. The equilibrium system of the economy can be summarized as

$$
\begin{aligned}
& \frac{\mathrm{d} K}{K}=\left[\alpha(1-g)-\frac{c}{n_{K} W}\right] \mathrm{d} t+\alpha(\mathrm{d} y-\mathrm{d} z)=\phi \mathrm{d} t+\alpha(\mathrm{d} y-\mathrm{d} z) \\
& \frac{\partial u(c, W)}{\partial c}=\left(1+\tau_{c}\right) X_{W} \\
& \left(r_{B} X_{W} W-\eta\right) \mathrm{d} t+\operatorname{cov}\left(\mathrm{d} w, \mathrm{~d} u_{B}\right) X_{W W} W^{2}=0 \\
& \left((1-\tau) r_{K} X_{W} W-\eta\right) \mathrm{d} t+\operatorname{cov}\left(\mathrm{d} w,\left(1-\tau^{\prime}\right) \mathrm{d} u_{K}\right) X_{W W} W^{2}=0 \\
& n_{B}+n_{K}=1
\end{aligned}
$$

and the transversality condition (TVC) plus the initial conditions.

Furthermore,

Proposition 3.3. The stochastic component of real rate of return on bonds, $\mathrm{d} u_{B}$, and total wealth, $\mathrm{d} w$, are determined by

$$
\begin{aligned}
& \mathrm{d} w=\alpha(\mathrm{d} y-\mathrm{d} z) \\
& \mathrm{d} u_{B}=\frac{\alpha}{n_{B}}\left[\left(1-n_{K}\left(1-\tau^{\prime}\right)\right) \mathrm{d} y-\mathrm{d} z\right]
\end{aligned}
$$

Proof. Using the intertemporal constancy of portfolio shares we have

$$
\frac{\mathrm{d} W}{W}=\frac{\mathrm{d} K}{K}=\frac{\mathrm{d} B}{B},
$$


i.e., all the real assets grow at a common stochastic rate. Combining with Eqs. (6), (15), (17), and (20), we get

$$
\begin{aligned}
\mathrm{d} w & =n_{B} \mathrm{~d} u_{B}+n_{K}\left(1-\tau^{\prime}\right) \alpha \mathrm{d} y=\alpha(\mathrm{d} y-\mathrm{d} z) \\
& =\frac{1}{n_{B}}\left[n_{B} \mathrm{~d} u_{B}+\alpha n_{K}\left(\mathrm{~d} z-\tau^{\prime} \mathrm{d} y\right)\right] .
\end{aligned}
$$

From the equations above, and noticing the fact $n_{B}+n_{K}=1$, it is easy to get $\mathrm{d} w$ and $\mathrm{d} u_{B}$.

These two equations enable us to compute all the necessary covariances and variances in the full equilibrium system. Eq. (19) implies that the stochastic shocks of government expenditure and production determine the stochastic rate of return on government bonds.

\section{An explicit example}

In order to find explicit solutions, we specify the utility function as in Bakshi and Chen (1996)

$$
u(c, W)=\frac{c^{1-\gamma}}{1-\gamma} W^{-\lambda},
$$

where $\gamma>0$, and $\lambda \geq 0$ when $\gamma \geq 1$, and $\lambda<0$ otherwise; $|\lambda|$ measures the investor's concern with his social status or measures his spirit of capitalism. The larger the parameter $|\lambda|$, the stronger the agent's spirit of capitalism or concern for social status.

Under the form of the utility function in (21), we have

Proposition 4.1. The first-order optimal conditions are

$$
\begin{aligned}
& \frac{c}{W}=\frac{\beta+\frac{1}{2} \sigma_{w}^{2}(1-\gamma-\lambda)(\gamma+\lambda)-\rho(1-\gamma-\lambda)}{\left(\gamma+\tau_{c}\right) /(1-\gamma)(1-\gamma-\lambda)}, \\
& \left(r_{B}-\frac{\eta}{\delta(1-\gamma-\lambda) W^{1-\gamma-\lambda}}\right) \mathrm{d} t=(\gamma+\lambda) \operatorname{cov}\left(\mathrm{d} w, \mathrm{~d} u_{B}\right), \\
& \left(r_{K}(1-\tau)-\frac{\eta}{\delta(1-\gamma-\lambda) W^{1-\gamma-\lambda}}\right) \mathrm{d} t=(\gamma+\lambda)\left(1-\tau^{\prime}\right) \operatorname{cov}\left(\mathrm{d} w, \mathrm{~d} u_{K}\right)
\end{aligned}
$$


where $\eta$ is the Lagrangian multiplier associated with constraint (7),

$$
\begin{aligned}
& \rho=n_{B} r_{B}+n_{K}(1-\tau) r_{K}, \\
& \mathrm{~d} w=n_{B} \mathrm{~d} u_{B}+n_{K}\left(1-\tau^{\prime}\right) \mathrm{d} u_{K}, \\
& \sigma_{w}^{2}=n_{B}^{2} \sigma_{B}^{2}+n_{K}^{2}\left(1-\tau^{\prime}\right)^{2} \sigma_{K}^{2}+2 n_{B} n_{K}\left(1-\tau^{\prime}\right) \sigma_{B K} .
\end{aligned}
$$

Eq. (9') gives the consumption-wealth ratio. For a logarithmic utility function in consumption, i.e., $\gamma=1$, we get $c / W=\beta$ : the consumption-wealth ratio is equal to the time discount rate. If $\gamma \neq 1$, then the effect of an increase in the expected net-of-tax return on the consumption-wealth ratio will be

$$
\frac{\mathrm{d}(c / W)}{\mathrm{d} \rho}=\frac{\gamma-1}{\gamma+\tau_{c}} .
$$

Therefore, an increase in the expected net-of-tax return $\rho$ will raise the consumption-wealth ratio if $\gamma>1$, and lower it otherwise. This can be explained as follows. When $\gamma>1$, the elasticity of intertemporal substitution, $1 / \gamma$, is relatively small. The representative agent will increase current consumption more than investment and wealth. On the other hand, when $\gamma<1$, the elasticity of intertemporal substitution is relatively large, and the agent will increase wealth holding more than consumption.

Similar analysis holds for the effect of the variance of wealth, $\sigma_{w}^{2}$, on $c / W$ :

$$
\frac{\mathrm{d}(c / w)}{\mathrm{d} \sigma_{w}^{2}}=\frac{1-\gamma}{2\left(\gamma+\tau_{c}\right)(\gamma+\lambda)}
$$

Therefore, an increase in the variance of wealth reduces the consumption-wealth ratio when $\gamma<1$, and increases the ratio when $\gamma>1$.

Eqs. $\left(10^{\prime}\right)$ and $\left(11^{\prime}\right)$ illustrate the asset pricing relationships. The term of $\eta / \delta(1-\gamma-\lambda) W^{1-\gamma-\lambda}$ can be regarded as 'risk-free' return in this all risky world - both returns on bonds and capital are uncertain. Eq. (10') implies that the return on bonds is equal to the 'risk-free' return plus a risk premium, which is proportional to the covariance between total wealth and risky bonds. Similarly, in Eq. $\left(11^{\prime}\right)$, for the net return on the risky capital, it is also equal to the 'risk-free' return plus a risk premium, which is proportional to the covariance between total wealth and risky capital.

Since $\rho$ is still endogenous in terms of holding shares for the two assets, we now use the full equilibrium system to derive explicit solutions to $c / W, n_{B}, n_{K}$, $r_{B}$, and $\phi$. With Proposition 3.3, and from the optimal conditions (10') and (11') 
plus Eq. (15), we have

$$
\begin{aligned}
& \sigma_{w}^{2}=\alpha^{2}\left(\sigma_{y}^{2}+\sigma_{z}^{2}\right) \mathrm{d} t, \\
& \operatorname{cov}\left(\mathrm{d} w, \mathrm{~d} u_{B}\right)=\frac{\alpha^{2}}{n_{B}}\left[\left(1-n_{K}\left(1-\tau^{\prime}\right)\right) \sigma_{y}^{2}+\sigma_{z}^{2}\right] \mathrm{d} t, \\
& \operatorname{cov}\left(\mathrm{d} w,\left(1-\tau^{\prime}\right) \mathrm{d} u_{K}\right)=\alpha^{2}\left(1-\tau^{\prime}\right) \sigma_{y}^{2} \mathrm{~d} t
\end{aligned}
$$

and

Proposition 4.2. The mean return on bonds and the stochastic growth rate of the economy are

$$
\begin{aligned}
& r_{B}=\alpha(1-\tau)+\frac{\gamma+\lambda}{n_{B}} \alpha^{2}\left(\sigma_{y}^{2}-\left(1-\tau^{\prime}\right) n_{K} \sigma_{y}^{2}-\left(1-\tau^{\prime}\right) n_{B} \sigma_{y}^{2}+\sigma_{z}^{2}\right), \\
& \phi=\frac{r_{B} n_{B}+(g-\tau) \alpha n_{K}+\tau_{c}(c / W)}{n_{B}}=\rho-\left(1+\tau_{c}\right) \frac{c}{W} .
\end{aligned}
$$

The first term on the right-hand side of Eq. (25) is the net (after-tax) return on capital, which is the same as in Turnovsky (1995); the second term on the right-hand side is the stochastic component of the return on bonds.

With Proposition 4.2, we now have our main theorem of this section:

Theorem 4.3. The explicit solutions of the economic system are

$$
\begin{gathered}
\frac{c}{W}=\frac{\beta}{\left(\gamma+\tau_{c}\right)(1-\gamma-\lambda) /(1-\gamma)} \\
-\frac{\alpha(1-\tau)+\frac{1}{2}(\gamma+\lambda) \alpha^{2}\left(\left(2 \tau^{\prime}-1\right) \sigma_{y}^{2}+\sigma_{z}^{2}\right)}{\left(\gamma+\tau_{c}\right) /(1-\gamma)}, \\
\phi=\alpha(1-\tau)+\alpha^{2}(\gamma+\lambda)\left(\tau^{\prime} \sigma_{y}^{2}+\sigma_{z}^{2}\right)-\left(1+\tau_{c}\right) \frac{c}{W}, \\
n_{K}=\frac{c / W}{\alpha(\tau-g)+\left(1+\tau_{c}\right) c / W-\alpha^{2}(\gamma+\lambda)\left(\tau^{\prime} \sigma_{y}^{2}+\sigma_{z}^{2}\right)} \\
n_{B}=1-n_{K}
\end{gathered}
$$

and the TVC

$$
\lim _{t \rightarrow \infty} E\left(W^{1-\gamma-\lambda} \mathrm{e}^{-\beta t}\right)=0
$$


Proof. Notice the conditions

$$
\begin{aligned}
& n_{B}+n_{K}=1, \\
& \rho=n_{B} r_{B}+n_{K} r_{K}(1-\tau), \\
& r_{B}=\alpha(1-\tau)+\frac{\gamma+\lambda}{n_{B}} \alpha^{2}\left(\left[\sigma_{y}^{2}-\left(1-\tau^{\prime}\right) n_{K} \sigma_{y}^{2}-\left(1-\tau^{\prime}\right) n_{B} \sigma_{y}^{2}\right]+\sigma_{z}^{2}\right) .
\end{aligned}
$$

We obtain

$$
\rho=\alpha(1-\tau)+\alpha^{2}(\gamma+\lambda)\left(\tau^{\prime} \sigma_{y}^{2}+\sigma_{z}^{2}\right)
$$

Thus, we have Eqs. (27) and (28). With Eq. (17), we have

$$
\phi=\alpha(1-g)-\frac{c}{n_{K} W}
$$

and Eq. (29).

Using Eqs. (25), (26), and the portfolio-selection constraint $n_{B}+n_{K}=1$, we have Eq. (30).

Please also note that the transversality condition (31) can be shown to be equivalent to $c / W>0$. In fact, since

$$
\mathrm{d} W=\phi W \mathrm{~d} t+W \mathrm{~d} w
$$

we have

$$
W(t)=W(0) \mathrm{e}^{\left(\phi-[(\gamma+\lambda) / 2] \sigma_{w}^{2}\right) t+w(t)-w(0)} .
$$

The TVC will be met if and only if

$$
(1-\gamma-\lambda)\left(\phi-\frac{\gamma+\lambda}{2} \sigma_{w}^{2}\right)-\beta<0 .
$$

By Eq. (27), we have

$$
(1-\gamma-\lambda)\left(\alpha(1-\tau)+\frac{\gamma+\lambda}{2} \alpha^{2}\left(\left(2 \tau^{\prime}-1\right) \sigma_{y}^{2}+\sigma_{z}^{2}\right)\right)<\beta .
$$

Eq. $\left(32^{\prime}\right)$ is just the condition for a positive consumption-wealth ratio.

\section{Comparative dynamics}

Now, we discuss how stochastic shocks (in production and government spending) and government fiscal policies affect the equilibrium. 


\subsection{Effects of stochastic shocks}

Differentiating with respect to $\sigma_{z}^{2}$ and $\sigma_{y}^{2}$, respectively, in Eq. (27), we have for $\gamma>1$

$$
\begin{aligned}
& \frac{\partial c / W}{\partial \sigma_{z}^{2}}=-\frac{(1-\gamma)(\gamma+\lambda) \alpha^{2}}{2\left(\tau_{c}+\gamma\right)}>0, \\
& \frac{\partial c / W}{\partial \sigma_{y}^{2}}=-\frac{(1-\gamma)(\gamma+\lambda)\left(2 \tau^{\prime}-1\right) \alpha^{2}}{2\left(\tau_{c}+\gamma\right)}<0 .
\end{aligned}
$$

Therefore, when the intertemporal elasticity of substitution is relatively small, a higher variance in government expenditure increases the consumption-wealth ratio, whereas the stochastic shock in production lowers the consumptionwealth ratio.

On the other hand, when $\gamma<1$, we have just the opposite results, namely,

$$
\begin{aligned}
& \frac{\partial c / W}{\partial \sigma_{z}^{2}}=-\frac{(1-\gamma)(\gamma+\lambda) \alpha^{2}}{2\left(\tau_{c}+\gamma\right)}<0, \\
& \frac{\partial c / W}{\partial \sigma_{y}^{2}}=-\frac{(1-\gamma)(\gamma+\lambda)\left(2 \tau^{\prime}-1\right) \alpha^{2}}{2\left(\tau_{c}+\gamma\right)}>0 .
\end{aligned}
$$

From Eq. (28), the equilibrium growth rate, $\phi$, varies with the stochastic shocks of government spending as follows. For all values of $\gamma$,

$$
\frac{\partial \phi}{\partial \sigma_{z}^{2}}=\frac{\alpha^{2}(\gamma+\lambda)\left(1+3 \tau_{c}+\gamma\left(1-\tau_{c}\right)\right)}{2\left(\gamma+\tau_{c}\right)}>0
$$

because $(\gamma+\lambda)>0$. Therefore, more volatility in government spending always increases the rate of economic growth. This is true because an increase in $\sigma_{z}^{2}$ raises the risk of bonds. The agent reduces his holding of government bonds and invests more in capital, which in turn leads to more output growth.

But for the shocks to the productivity, the mean growth rate of the economy can increase or decrease depending on the values of $\gamma$ and other parameters. For example, when $\gamma>1$, and $\tau^{\prime}<50 \%$,

$$
\frac{\partial \phi}{\partial \sigma_{y}^{2}}=\alpha^{2}\left[\tau^{\prime}+\frac{\left(2 \tau^{\prime}-1\right)\left(1+\tau_{c}\right)(1-\gamma)}{2\left(\gamma+\tau_{c}\right)}\right](\gamma+\lambda)<0 .
$$

In this case, an increase in the variance of the productivity shocks lowers the growth rate. But when $\gamma<1, \partial \phi / \partial \sigma_{y}^{2}$ has an ambiguous sign. Our results confirm the complicated pictures of the effects of stochastic shocks on output growth in Obstfeld (1994), Turnovsky (1995), and Grinols and Turnovsky (1996). 
The dependence of the shares of asset holding on the stochastic shocks can be derived from Eq. (29):

$$
\begin{aligned}
& \frac{\partial n_{K}}{\partial \sigma_{z}^{2}}=n_{K}\left\{\frac{\left[1-n_{K}\left(1+\tau_{c}\right)\right] \partial(c / W) / \partial \sigma_{z}^{2}}{c / W}+\alpha^{2}(\gamma+\lambda) \frac{n_{k}}{c / W}\right\}>0, \\
& \frac{\partial n_{K}}{\partial \sigma_{y}^{2}}=n_{K}\left\{\frac{\left[1-n_{K}\left(1+\tau_{c}\right)\right] \partial(c / W) / \partial \sigma_{y}^{2}}{c / W}+\frac{n_{K}}{c / W} \alpha^{2}(\gamma+\lambda) \tau^{\prime}\right\} .
\end{aligned}
$$

The first equation above tells us that the stochastic shock in government expenditure will enhance the holding of risky capital. In the second equation the effect of the stochastic shock in production on the holding of risky capital is ambiguous.

We have derived the value function $X(W, t)$ in Appendix B. Let $W(0)$ denote the initial stock of wealth. We have the following welfare function:

$$
X(W(0))=\delta W(0)^{1-\gamma-\lambda}
$$

where

$$
\delta=\frac{1}{\left(1+\tau_{c}\right)(1-\gamma-\lambda)}\left(\frac{c}{W}\right)^{-\gamma} .
$$

However, $W(0)$ is itself endogenously determined by

$$
W(0)=\frac{K_{0}}{n_{K}} .
$$

Therefore, with some simple manipulations, welfare is given by

$$
X\left(K_{0}\right)=n_{K}^{\gamma+\lambda-1} \frac{1}{\left(1+\tau_{c}\right)(1-\gamma-\lambda)}\left(\frac{c}{W}\right)^{-\gamma} K_{0}^{1-\gamma-\lambda},
$$

where $c / W$ and $n_{K}$ are determined as in Theorem 1 . Taking differentiation in Eq. (35), we get

$$
\frac{\mathrm{d} X}{X}=(\gamma+\lambda-1) \frac{\mathrm{d} n_{K}}{n_{K}}-\gamma \frac{\mathrm{d}(c / W)}{c / W} .
$$

Now, we have

$$
\begin{aligned}
& \frac{\partial X}{\partial \sigma_{z}^{2}}=(\gamma+\lambda-1) \frac{X \partial n_{K} / \partial \sigma_{z}^{2}}{n_{K}}-\gamma \frac{X \partial(c / W) / \partial \sigma_{z}^{2}}{c / W}, \\
& \frac{\partial X}{\partial \sigma_{y}^{2}}=(\gamma+\lambda-1) \frac{X \partial n_{K} / \partial \sigma_{y}^{2}}{n_{K}}-\gamma \frac{X \partial(c / W) / \partial \sigma_{y}^{2}}{c / W} .
\end{aligned}
$$


These equations imply that the effects on welfare of the stochastic shocks in government expenditure and production are ambiguous.

\subsection{Effects of fiscal policies}

Now, we turn to how taxes on capital income and consumption impact on the equilibrium.

First, differentiating all endogenous variables with respect to the tax on the deterministic part of capital income, $\tau$, in Eqs. (27)-(29), we have

$$
\begin{aligned}
& \frac{\mathrm{d}(c / W)}{\mathrm{d} \tau}=\frac{\alpha(1-\gamma)}{\gamma+\tau_{c}}, \\
& \frac{\mathrm{d} \phi}{\mathrm{d} \tau}=-\frac{\alpha\left(1-\gamma \tau_{c}+2 \tau_{c}\right)}{\gamma+\tau_{c}}, \\
& \frac{\mathrm{d} n_{K}}{\mathrm{~d} \tau}=n_{K}\left\{\frac{\left(1-n_{K}\left(1+\tau_{c}\right)\right) \partial(c / W) / \partial \tau}{c / W}-\alpha \frac{n_{K}}{c / W}\right\}, \\
& \frac{\mathrm{d} X}{\mathrm{~d} \tau}=X\left\{\frac{(\gamma+\lambda-1) \partial n_{K} / \partial \tau}{n_{K}}-\gamma \frac{\partial(c / W) / \partial \tau}{c / W}\right\} .
\end{aligned}
$$

If $\gamma=1, c / W$ is independent of the tax rate, because in this case $c / W=\beta$, which is independent of $\tau$. When $0<\gamma<1$, we notice that a rise in the taxation on the deterministic component of capital income has an ambiguous effect on welfare. But, it is clear that

$$
\frac{\mathrm{d}(c / W)}{\mathrm{d} \tau}>0, \quad \frac{\mathrm{d} \phi}{\mathrm{d} \tau}<0
$$

Therefore, a higher tax on the deterministic component of capital income will increase the consumption-wealth ratio and decrease the economic growth rate. This can be explained as follows: a higher tax on capital income will lower the return on capital. As the agent switches away from capital to bonds and consumption, this reduces capital accumulation, lowers the growth rate, and increases the consumption-wealth ratio.

When $\gamma>1$, we still find that capital income taxation reduces the holding share of risky capital and lowers the growth rate

$$
\frac{\mathrm{d} \phi}{\mathrm{d} \tau}<0, \quad \frac{\mathrm{d} n_{K}}{\mathrm{~d} \tau}<0 .
$$

But it reduces the consumption-wealth ratio: $\mathrm{d}(c / W) / \mathrm{d} \tau<0$. 
Second, we look at the effects on the equilibrium of the tax on the stochastic component of capital income:

$$
\begin{aligned}
& \frac{\partial(c / W)}{\partial \tau^{\prime}}=-\frac{\alpha^{2}(\gamma+\lambda)(1-\gamma) \sigma_{y}^{2}}{\gamma+\tau_{c}}, \\
& \frac{\partial \phi}{\partial \tau^{\prime}}=\alpha^{2}(\gamma+\lambda) \sigma_{y}^{2} \frac{1-\gamma \tau_{c}+2 \tau_{c}}{\gamma+\tau_{c}}, \\
& \frac{\partial n_{K}}{\partial \tau^{\prime}}=n_{K} \frac{1-n_{K}\left(1+\tau_{c}\right)}{c / W} \frac{\partial(c / W)}{\partial \tau^{\prime}}+\alpha^{2}(\gamma+\lambda) \sigma_{y}^{2} \frac{n_{K}^{2}}{c / W}, \\
& \frac{\partial X}{\partial \tau^{\prime}}=X(\gamma+\lambda-1) \frac{\partial n_{K} / \partial \tau^{\prime}}{n_{K}}-\gamma \frac{X \partial(c / W) / \partial \tau^{\prime}}{c / W} .
\end{aligned}
$$

These results are very similar to the ones for the tax on the deterministic component of capital income. Still,

$$
\frac{\partial(c / W)}{\partial \tau^{\prime}}<0, \quad \frac{\partial \phi}{\partial \tau^{\prime}}>0
$$

when $0<\gamma<1$; and

$$
\frac{\partial(c / W)}{\partial \tau^{\prime}}>0, \quad \frac{\partial \phi}{\partial \tau^{\prime}}>0, \quad \frac{\partial n_{k}}{\partial \tau^{\prime}}>0
$$

when $\gamma>1$.

From the analysis above, the relationship between income taxes and growth is similar to the one in Turnovsky (1995): Raising the tax rate on the stochastic component of capital income has the opposite effect to raising the tax rate on the deterministic component of income.

Finally, we examine the effects of the consumption tax on the equilibrium. Recall that from the Ramsey-Cass-Koopmans model, the consumption tax does not affect the rate of economic growth and long-run capital accumulation. In the long run, it only crowds out private consumption. Here we have

$$
\frac{\partial(c / W)}{\partial \tau_{c}}=-\frac{c / W}{\gamma+\tau_{c}}<0 .
$$

That is to say, increasing the consumption tax will reduce the consumption-wealth ratio because a higher consumption tax decreases private consumption directly, and the agent has more money to invest in capital and bonds, which in turn increases wealth. Therefore, the consumption-wealth ratio decreases as a result of a higher consumption tax. 
For the growth rate, a rise in the consumption tax results in

$$
\frac{\partial \phi}{\partial \tau_{c}}=\frac{c / W(1-\gamma)}{\gamma+2 \tau_{c}} .
$$

Hence, $\partial \phi / \partial \tau_{c}<0$ when $\gamma>1$; and $\partial \phi / \partial \tau_{c}>0$ when $0<\gamma<1$. Because when $\gamma>1$, the elasticity of intertemporal substitution is small, and the agent is less willing to sacrifice current consumption for future consumption. Therefore, the cut in investment is more than the cut in current consumption, which leads to a lower long-run growth rate. On the other hand, when the elasticity of intertemporal substitution is relatively large (i.e. $0<\gamma<1$ ), the agent is more willing to give up current consumption for investment in risky capital. As a result, a higher consumption tax leads to a higher growth rate.

As for welfare, we have

$$
\begin{aligned}
\frac{\partial X}{\partial \tau_{c}} & =(\gamma+\lambda-1) \frac{X \partial n_{K} / \partial \tau_{c}}{n_{K}}-\gamma \frac{X \partial(c / W) / \partial \tau_{c}}{c / W}-\frac{X}{1+\tau_{c}} \\
& =\frac{X(1-\gamma)(\gamma+\lambda-1)\left(1+\tau_{c}\right) n_{K}-\lambda\left(1+\tau_{c}\right)+(1-\gamma)}{\left(\gamma+\tau_{c}\right)\left(1+\tau_{c}\right)} .
\end{aligned}
$$

Therefore, $\partial X / \partial \tau_{c}<0$ when $\gamma>1$. The explanation is simple. Since the elasticity of intertemporal substitution is small, current consumption will not be severely cut as a result of a consumption tax, whereas current investment in assets is reduced. In the long run, the agent will accumulate less assets and earn less income. His consumption and asset holdings are all reduced in the long run. Since welfare is defined on both consumption and wealth accumulation, his long-run welfare is also lower. For $\gamma<1$, the welfare effect of a consumption tax is ambiguous because the direct effect of a higher consumption tax reduces consumption. But with a larger elasticity of intertemporal substitution the agent may increase his asset holdings, which in turn can lead to more asset accumulation and more income. This rising income can give rise to more long-run consumption. Again since the agent's welfare is defined on both consumption and asset holdings, his welfare may also rise in this case.

\section{Effects of the spirit of capitalism}

In this section, we will discuss how the spirit of capitalism or the concern for social status affects asset pricing and economic growth. For simplicity, we set consumption tax, $\tau_{c}$, to zero in this section.

First, we give the equilibrium asset-pricing relationships. Following Turnovsky (1995), we define the market portfolio as $Q=n_{B} W+n_{K} W$, and 
the return rate on the market portfolio as

$$
\begin{aligned}
r_{Q} & \equiv \rho=r_{B} n_{B}+r_{K}(1-\tau) n_{K} \\
& =\alpha(1-\tau)+(\gamma+\lambda) \alpha^{2}\left(\tau^{\prime} \sigma_{y}^{2}+\sigma_{z}^{2}\right) .
\end{aligned}
$$

Now we have

Proposition 6.1. The equilibrium asset-pricing relationships are

$$
r_{i}-\frac{\eta}{\delta(1-\gamma-\lambda) W^{1-\gamma-\lambda}}=\beta_{i}\left(r_{Q}-\frac{\eta}{\delta(1-\gamma-\lambda) W^{1-\gamma-\lambda}}\right),
$$

where $i=B, K$,

$$
\begin{aligned}
& \beta_{B}=\frac{\operatorname{cov}\left(\mathrm{d} w, \mathrm{~d} u_{B}\right)}{\operatorname{var}(\mathrm{d} w)}=\frac{\left(1-n_{K}\left(1-\tau^{\prime}\right)\right) \sigma_{y}^{2}+\sigma_{z}^{2}}{n_{B}\left(\sigma_{y}^{2}+\sigma_{z}^{2}\right)}, \\
& \beta_{K}=\frac{\operatorname{cov}\left(\mathrm{d} w, \mathrm{~d} u_{K}\right)}{\operatorname{var}(\mathrm{d} w)}=\frac{\left(1-\tau^{\prime}\right) \sigma_{y}^{2}}{\sigma_{y}^{2}+\sigma_{z}^{2}} .
\end{aligned}
$$

Proof. From

$$
\frac{\eta}{\delta(1-\gamma-\lambda) W^{1-\gamma-\lambda}}=\alpha(1-\tau)-(\gamma+\lambda) \alpha^{2}\left(1-\tau^{\prime}\right) \sigma_{y}^{2}
$$

and

$$
r_{Q}=\alpha(1-\tau)+(\gamma+\lambda) \alpha^{2}\left(\tau^{\prime} \sigma_{y}^{2}+\sigma_{z}^{2}\right),
$$

we obtain

$$
r_{Q}-\frac{\eta}{\delta(1-\gamma-\lambda) W^{1-\gamma-\lambda}}=(\gamma+\lambda) \alpha^{2}\left(\sigma_{y}^{2}+\sigma_{z}^{2}\right)
$$

and using Proposition 4.1, we get the result.

Again $\eta / \delta(1-\gamma-\lambda) W^{1-\gamma-\lambda}$ can be regarded as the risk-free return. Eq. (42) indicates that the returns on risky assets (government bonds and capital) are given by the familiar consumption-based capital asset pricing model with $r_{Q}$ as the return on the market portfolio.

Furthermore, if we define the return on the market portfolio in the absence of the spirit of capitalism as $\bar{r}_{Q}$, then, in our definition of the return of the market portfolio $r_{Q}$, we set $\lambda=0$. Hence,

$$
\bar{r}_{Q}=\alpha(1-\tau)+\gamma \alpha^{2}\left(\tau^{\prime} \sigma_{y}^{2}+\sigma_{z}^{2}\right) .
$$


This is just the return on the market portfolio in Turnovsky (1995). At the same time, we have

$$
\frac{\eta}{\delta(1-\gamma) W^{1-\gamma}}=\alpha(1-\tau)-\gamma \alpha^{2}\left(1-\tau^{\prime}\right) \sigma_{y}^{2}
$$

Hence, we obtain the asset-pricing relationships as

$$
\bar{r}_{i}-\frac{\eta}{\delta(1-\gamma) W^{1-\gamma}}=\beta_{i}\left(\bar{r}_{Q}-\frac{\eta}{\delta(1-\gamma) W^{1-\gamma}}\right) .
$$

Because $\bar{r}_{Q}<r_{Q}$, simple calculations yield

$$
r_{i}-\frac{\eta}{\delta(1-\gamma-\lambda) W^{1-\gamma-\lambda}}>\bar{r}_{i}-\frac{\eta}{\delta(1-\gamma) W^{1-\gamma}}
$$

Eq. (43) implies that, with the spirit of capitalism, the gap between the returns on risky assets and the return on the risk-free asset will be enlarged. Like Bakshi and Chen (1996), our findings can be used to partially explain the equity premium puzzle in Mehra and Prescott (1985).

For the growth rate, social welfare, and portfolio selection, we have

Proposition 6.2. The effects of the spirit of capitalism on $c / W, n_{K}, \phi$, and $X$ are as follows:

$$
\begin{aligned}
\frac{\partial(c / W)}{\partial \lambda}= & \frac{(1-\gamma)}{2 \gamma} \alpha^{2}\left(\left(1-2 \tau^{\prime}\right) \sigma_{y}^{2}-\sigma_{z}^{2}\right)+\frac{\beta(1-\gamma)}{\gamma(1-\gamma-\lambda)^{2}} \\
& =\frac{(1-\gamma)}{2 \gamma} \alpha^{2}\left(\sigma_{y}^{2}+\sigma_{z}^{2}\right)+\frac{\beta(1-\gamma)}{\gamma(1-\gamma-\lambda)^{2}}+\frac{1-\gamma}{\gamma} \alpha^{2}\left(\tau^{\prime} \sigma_{y}^{2}+\sigma_{z}^{2}\right) \\
\frac{\partial \phi}{\partial \lambda}=- & \frac{(1-\gamma)}{2 \gamma} \alpha^{2}\left(\sigma_{y}^{2}+\sigma_{z}^{2}\right)-\frac{\beta(1-\gamma)}{\gamma(1-\gamma-\lambda)^{2}}+\frac{1}{\gamma} \alpha^{2}\left(\tau^{\prime} \sigma_{y}^{2}+\sigma_{z}^{2}\right) \\
\frac{\partial n_{K}}{\partial \lambda}= & \frac{n_{K}}{c / W}\left[\left(1-n_{K}\right)\left(\frac{(1-\gamma)}{2 \gamma} \alpha^{2}\left(\sigma_{y}^{2}+\sigma_{z}^{2}\right)+\frac{\beta(1-\gamma)}{\gamma(1-\gamma-\lambda)^{2}}\right)\right. \\
& -\frac{n_{K}}{c / W} \frac{1-\gamma-n_{K}}{\gamma} \alpha^{2}\left(\tau^{\prime} \sigma_{y}^{2}+\sigma_{z}^{2}\right) \\
\frac{\partial X}{\partial \lambda}= & (\gamma+\lambda-1) \frac{X \partial n_{K} / \partial \lambda}{n_{K}}-\gamma \frac{X \partial(c / W) / \partial \lambda}{c / W}
\end{aligned}
$$


If $\gamma>1$, we have $\lambda>0$. Then

$$
\frac{\partial \phi}{\partial \lambda}>0, \quad \frac{\partial n_{K}}{\partial \lambda}>0
$$

Similarly, if $\gamma<1$, we have $\lambda<0$. In this case $|\lambda|$ measures the spirit of capitalism, and

$$
\frac{\partial \phi}{\partial(-\lambda)}>0, \quad \frac{\partial n_{K}}{\partial(-\lambda)}>0 .
$$

Therefore, an increase in the spirit of capitalism will always increase the growth rate and the holding share of risky capital. With a strong spirit of capitalism, the agent cares more about his social status and the power of wealth, and will accumulate more wealth and take more risk in investment in order to improve his social status.

If we further impose the condition that $\tau^{\prime}<\frac{1}{2}\left(1-\sigma_{z}^{2} / \sigma_{y}^{2}\right)$, then

$$
\frac{\partial c / W}{\partial \lambda}<0, \quad \frac{\partial X}{\partial \lambda}>0
$$

when $\gamma>1$ and $\lambda>0$. Furthermore, with the same condition on the tax rate on the stochastic component of capital income, i.e., $\tau^{\prime}<\frac{1}{2}\left(1-\sigma_{z}^{2} / \sigma_{y}^{2}\right)$,

$$
\frac{\partial c / W}{\partial(-\lambda)}<0, \quad \frac{\partial X}{\partial(-\lambda)}>0
$$

when $\gamma<1$ and $\lambda<0$. Given the assumption on the tax rate, a strong spirit of capitalism always reduces the consumption-wealth ratio. Since the agent's utility is defined on both consumption and wealth accumulation, his long-run welfare rises as a result of higher wealth and possibly even higher consumption.

\section{Conclusion}

In this paper, we have extended the existing frameworks of stochastic growth and asset pricing by including the spirit of capitalism and concern for social status - direct preferences for wealth. In this extended model, we have studied how stochastic shocks in production and government spending affect consumption, wealth accumulation, economic growth, and welfare. This paper has further extended the studies by Eaton, Grinols, Obstfeld, and Turnovsky, among others, to consider the impact of various taxes on the consumptionwealth ratio, growth, and welfare. The existence of the positive effect of a consumption tax on growth is a result of the spirit-of-capitalism or wealth-is-status 
model. Without the spirit of capitalism, a consumption tax has no effect on output growth.

The direct effect of the spirit of capitalism on the economy has been also explicitly considered in this paper. It is shown that the existence of the spirit of capitalism can better explain the difference between the rates of return on government bonds and risky stock - the Mehra-Prescott risk-premium puzzle. In the spirit-of-capitalism or wealth-is-status model, the gap between the returns on risky assets and the risk-free asset is always larger. Furthermore, a higher spirit of capitalism or a stronger concern for social status can lead to higher output growth, more holdings of risky capital, and a lower consumption-wealth ratio.

This research can be extended in many directions. The discussion of monetary policy is a natural choice when wealth includes bonds, capital, and real balances. The results can be compared to the ones in Turnovsky (1995), Grinols (1996), Turnovsky and Grinols (1996) where only real balances are included in the utility function. Another interesting extension is to examine the optimal (welfare-maximizing) choices of capital income taxes and consumption tax following Corsetti (1992, 1997), Turnovsky (1995), and Turnovsky and Grinols (1996).

\section{Appendix A}

Consider the optimization problem:

$$
\max E \int_{0}^{\infty} u\left(c_{t}, W_{t}\right) \mathrm{e}^{-\beta t} \mathrm{~d} t
$$

subject to

$$
\frac{\mathrm{d} W}{W}=\left(\rho-\left(1+\tau_{c}\right) \frac{c_{t}}{W_{t}}\right) \mathrm{d} t+\mathrm{d} w,
$$

where

$$
\begin{aligned}
& \rho=n_{B} r_{B}+n_{K}(1-\tau) r_{K}, \\
& \mathrm{~d} w=n_{B} \mathrm{~d} u_{B}+n_{K}\left(1-\tau^{\prime}\right) \mathrm{d} u_{K} .
\end{aligned}
$$

From Eq. (A.4), we have

$$
\sigma_{w}^{2}=n_{B}^{2} \sigma_{B}^{2}+n_{K}^{2}\left(1-\tau^{\prime}\right)^{2} \sigma_{K}^{2}+2 n_{B} n_{K}\left(1-\tau^{\prime}\right) \sigma_{B K} .
$$

To solve the problem, we define the value function $V(W, t)$

$$
V(W, t)=\mathrm{e}^{-\beta t} X(W, t)
$$


and formally, it is also defined

$$
L_{W}(V(W, t))=\frac{\partial V}{\partial t}+\left(\rho-\left(1+\tau_{c}\right) \frac{c_{t}}{W_{t}}\right) W \frac{\partial V}{\partial W}+\frac{1}{2} \sigma_{w}^{2} W^{2} \frac{\partial^{2} V}{\partial W^{2}}
$$

The Lagrangian function associated with the problem is

$$
\mathrm{e}^{-\beta t} u\left(c_{t}, W_{t}\right)+L_{W}\left(\mathrm{e}^{-\beta t} X(W, t)\right)+\mathrm{e}^{-\beta t} \eta\left(1-n_{B}-n_{K}\right)
$$

In this case, the corresponding first-order conditions for maximization are

$$
\begin{aligned}
& \frac{\partial u(c, W)}{\partial c}=\left(1+\tau_{c}\right) X_{W}, \\
& \left(r_{B} X_{W} W-\eta\right) \mathrm{d} t+\operatorname{cov}\left(\mathrm{d} w, \mathrm{~d} u_{B}\right) X_{W W} W^{2}=0, \\
& \left((1-\tau) r_{K} X_{W} W-\eta\right) \mathrm{d} t+\operatorname{cov}\left(\mathrm{d} w,\left(1-\tau^{\prime}\right) \mathrm{d} u_{K}\right) X_{W W} W^{2}=0, \\
& n_{B}+n_{K}=1 .
\end{aligned}
$$

These equations determine the optimal choices of $c / W, n_{B}, n_{K}$, and $\eta$ as the functions of $X_{W}$ and $X_{W W}$. Furthermore, the value function must satisfy the Bellman equation

$$
\begin{aligned}
& u(c, W)-\beta X(W, t)+X_{t}(W, t)+\left(\rho-\left(1+\tau_{c}\right) \frac{c}{W}\right) W X_{W} \\
& +\frac{1}{2} \sigma_{w}^{2} W^{2} X_{W W}=0
\end{aligned}
$$

Now, we have completed the proof of Proposition 3.1.

\section{Appendix B}

To show Proposition 4.1, we rewrite the utility function here:

$$
u(c, W)=\frac{c^{1-\gamma}}{1-\gamma} W^{-\lambda}
$$


The form of the value function is postulated as

$$
X(W, t)=\delta W^{1-\gamma-\lambda},
$$

where $\delta$ is to be determined.

Differentiating with respect to $W$ yields

$$
X_{W}=\delta(1-\gamma-\lambda) W^{-\gamma-\lambda}, \quad X_{W W}=\delta(1-\gamma-\lambda)(-\gamma-\lambda) W^{-\gamma-\lambda-1} .
$$

Now the corresponding first-order conditions are

$$
\begin{aligned}
& \frac{c}{W}=\left(\left(1+\tau_{c}\right) \delta(1-\gamma-\lambda)\right)^{-1 / \gamma} \\
& \left(r_{B} \delta(1-\gamma-\lambda) W^{1-\gamma-\lambda}-\eta\right) \mathrm{d} t \\
& \quad+\operatorname{cov}\left(\mathrm{d} w, \mathrm{~d} u_{B}\right) \delta(1-\gamma-\lambda)(-\gamma-\lambda) W^{1-\gamma-\lambda}=0, \\
& \operatorname{cov}\left(\mathrm{d} w,\left(1-\tau^{\prime}\right) \mathrm{d} u_{K}\right) \delta(1-\gamma-\lambda)(-\gamma-\lambda) W^{1-\gamma-\lambda} \\
& \quad+\left((1-\tau) r_{K} \delta(1-\gamma-\lambda) W^{1-\gamma-\lambda}-\eta\right) \mathrm{d} t=0 .
\end{aligned}
$$

Substituting for $c$ in the Bellman equation (A.12) leads to

$$
\begin{aligned}
& \frac{\left(\left(1+\tau_{c}\right) \delta(1-\gamma-\lambda)\right)^{-(1-\gamma) / \gamma}}{1-\gamma}-\beta \delta \\
& +\delta(1-\gamma-\lambda)\left(\rho-\left(\left(1+\tau_{c}\right) \delta(1-\gamma-\lambda)\right)^{-1 / \gamma}\right) \\
& \quad+\frac{1}{2} \sigma_{w}^{2} \delta(1-\gamma-\lambda)(-\gamma-\lambda)=0
\end{aligned}
$$

or

$$
\left(\left(1+\tau_{c}\right) \delta(1-\lambda-\gamma)\right)^{-1 / \gamma}=\frac{\beta+\frac{1}{2} \sigma_{w}^{2}(1-\gamma-\lambda)(\gamma+\lambda)-\rho(1-\gamma-\lambda)}{\left(1+\tau_{c}\right)(1-\gamma-\lambda) /(1-\gamma)-(1-\gamma-\lambda)}
$$

Thus, we have obtained all the expressions in Proposition 4.1:

$$
\frac{c}{W}=\frac{\beta+\frac{1}{2} \sigma_{w}^{2}(1-\gamma-\lambda)(\gamma+\lambda)-\rho(1-\gamma-\lambda)}{\left(1+\tau_{c}\right)(1-\gamma-\lambda) /(1-\gamma)-(1-\gamma-\lambda)}
$$




$$
\begin{aligned}
& \left(r_{B}-\frac{\eta}{\delta(1-\gamma-\lambda) W^{1-\gamma-\lambda}}\right) \mathrm{d} t=(\gamma+\lambda) \operatorname{cov}\left(\mathrm{d} w, \mathrm{~d} u_{B}\right), \\
& \left(r_{K}(1-\tau)-\frac{\eta}{\delta(1-\gamma-\lambda) W^{1-\gamma-\lambda}}\right) \mathrm{d} t=(\gamma+\lambda) \operatorname{cov}\left(\mathrm{d} w, \mathrm{~d} u_{K}\left(1-\tau^{\prime}\right)\right) .
\end{aligned}
$$

\section{References}

Bakshi, G.S., Chen, Z., 1996. The spirit of capitalism and stock-market prices. American Economic Review 86, 133-157.

Cole, H., Mailath, G., Postlewaite, A., 1992. Social norms, saving behavior and growth. Journal of Political Economy 100, 1092-1125.

Cole, H., Mailath, G., Postlewaite, A., 1995. Incorporating concern for relative wealth into economic models. Quarterly Review (Federal Reserve Bank of Minneapolis) 19, 12-21.

Corsetti, G., 1992. A portfolio approach to endogenous growth: Eaton's model revisited. Center Discussion Paper No. 678, Economic Growth Center at Yale University.

Corsetti, G., 1997. A portfolio approach to endogenous growth: equilibrium and optimal policy. Journal of Economic Dynamics and Control 21, 1627-1644.

Duesenberry, J., 1948. Income, Saving and the Theory of Consumer Behavior. Harvard University Press, Cambridge, MA.

Eaton, J., 1981. Fiscal policy, inflation, and the accumulation of risk capital. Review of Economic Studies 48, 435-445.

Fershtman, C., Weiss, Y., 1993. Social status, culture and economic performance. Economic Journal 103, 946-959.

Fershtman, C., Murphy, K., Weiss, Y., 1996. Social status, education, and growth. Journal of Political Economy 106, 108-132.

Fischer, S., 1975. The demand for index bonds. Journal of Political Economy 83, 509-534.

Frank, R., 1985. Choosing the Right Pond: Human Behavior and the Quest for Status. Oxford University Press, Oxford.

Grinols, E., 1996. The link between domestic investment and domestic savings in open economies: evidence from balanced stochastic growth. Review of International Economics 4, 119-140.

Grinols, E., Turnovsky, S., 1993. Risk, the financial market, and macroeconomic equilibrium. Journal of Economic Dynamics and Control 17, 1-36.

Grinols, E., Turnovsky, S., 1994. Exchange rate determination and asset prices in a stochastic small open economy. Journal of International Economics 36, 75-97.

Grinols, E., Turnovsky, S., 1996. Optimal government finance policy and exchange rate management in a stochastically growing open economy. Journal of International Money and Finance 15, 687-716.

Keynes, J.M., 1971. The Economic Consequences of the Peace. St. Martin's Press, London.

Kurz, M., 1968. Optimal economic growth and wealth effects. International Economic Review 9, 348-357.

Mehra, R., Prescott, E.C., 1985. The equity premium: a puzzle. Journal of Monetary Economy 15, $145-161$.

Obstfeld, M., 1994. Risk-taking, global diversification, and growth. American Economic Review 84, 1310-1329.

Spence, M., 1974. Market Signaling: Information Transfer in Hiring and Related Screening Processes. Harvard University Press, Cambridge, MA. 
Turnovsky, S., 1993. Macroeconomic policies, growth, and welfare in a stochastic economy. International Economic Review 35, 953-981.

Turnovsky, S., 1995. Methods of Macroeconomic Dynamics. MIT Press, Cambridge, MA.

Weber, M., 1958. The Protestant Ethic and the Spirit of Capitalism. Charles Scribner's Sons, New York.

Zou, H., 1994. The spirit of capitalism and long-run growth. European Journal of Political Economy 10, 279-293.

Zou, H., 1995. The spirit of capitalism and savings behavior. Journal of Economic Behavior and Organization 28, 131-143. 